\title{
Wettability Changing of FINEMET Substrates Using High-Energy Femtosecond Laser Impulses
}

\begin{abstract}
M. BERCZELI*
Department of Materials Technology, John von Neumann University, Kecskemét, Izsáki street 10, Hungary

FINEMET-type metallic glass ribbon was improved for better quality of joining technologies by reducing the wetting contact angle on the treated surface. The surface activation of the boundary layers was obtained with the femtosecond laser impulses. Using magnetic, wetting, topography and structure analyses three type of surface activated metallic glass specimens were investigated. The first type with invisible, the second with slightly noticeable, and the last with significant laser surface patch. The laser activation modified the surfaces to hydrophilic, while the magnetic properties were much less damaged. In this work, a wetting improvement laser method was developed without damaging the base material properties. The laser surface treatment increased the surface free energy of the metallic glass to create a hydrophilic surface, but the magnetic properties does not changed significantly.
\end{abstract}

DOI: 10.12693/APhysPolA.137.864

PACS/topics: metallic glass ribbon, magnetic properties, wettability, femtosecond laser

\section{Introduction}

FINEMET type materials are widely used because of excellent magnetic properties. Many improvements have been made to replace the parts of an electric motor with the ribbons, since the efficiency of the motor can increased by utilizing the magnetic properties [1]. To make the ribbons particularly useful in electric motors, isolation of each coil is extremely important. The best solution for this is the isolation with direct coating of the surfaces [2]. It is important to have good wetting properties in favor of good adhesion conditions between the coating and the ribbon. The surface treatment of metals can change the surface properties and the adhesion conditions [3]. In case of femtosecond laser beam activation it might drastically change the interface properties, thus resulting in an improvement of wetting contact angle that plays a major role in the development of bonding technologies. The interaction of the laser impulses on the material can modify the magnetic properties [4]. The only problem may be that the magnetic properties of the magnetic ribbon could be damaged under the laser treatment [5]. In this work, development of a technology is performed to improve the wettability, while the magnetic properties of the material do not change.

\section{Experimental}

Surface treatment was achieved on FINEMET-type metallic glass. Each specimen had a dimension of $100 \mathrm{~mm} \times 10 \mathrm{~mm} \times 0.028 \mathrm{~mm}$ (longitudinally directed), and 10 piece of surface activating hatched-circle were

\footnotetext{
* corresponding author; e-mail:

berczeli.miklos@gamf .uni-neumann.hu
}

made with diameter of $5 \mathrm{~mm}$, equal distance from each other, and $2.5 \mathrm{~mm}$ from the edges of the specimen. The laser treating process was performed using a Coherent Monaco Femtosecond Laser with wavelength of $1035 \mathrm{~nm}$. The laser impulses had 300 fs of width, the frequency $188 \mathrm{kHz}$, and the average power was set between $2 \mathrm{~W}$ and $20 \mathrm{~W}$ (i.e., from $5 \%$ to $50 \%$ power). The scanning speed was fixed to $5 \mathrm{~m} / \mathrm{sec}$. The beam was focused on the surface with a diameter of $80 \mu \mathrm{m}$. A protective atmosphere was assured with 6.0 Argon gas. The metallic glass specimens were cut after the surface treatment using the same laser device. The wettability properties were measured with a contact angle measurement system, and the test uses $5 \mu \mathrm{l}$ distilled water and $5 \mu \mathrm{l}$ ethylene glycol droplet. The topography changing effect of the laser beam was investigated using a Zeiss Axio Imager 2 optical, and a Zeiss EVO scanning electron microscope. For the differential scanning calorimetry test a TA Q200 DSC devices was used with a heating speed of $10 \mathrm{~K} / \mathrm{min}$, where each specimen was $15 \mathrm{mg}$. In order to measure the magnetic changing effect of the laser surface activation magnetometer measurement was examined according to L. Novak et al [6].

\section{Results and discussion}

\subsection{The wettability results of the treated surfaces.}

To investigate the effect of average power of the femtosecond laser on the wetting property, the scanning speed, the hatch distance, and the diameter of laser beam were fixed at values $5 \mathrm{~m} / \mathrm{s}, 50 \mu \mathrm{m}$, and $80 \mu \mathrm{m}$. Results of the femtosecond laser surface activation effect on changing the contact angle, can be seen in Fig. 1.

The power higher than $50 \%$ had not been used because of the laser thermal effects which mostly change the microstructure of the metallic glass to rigid and unacceptable. In turn, as the laser power is lower, e.g., $5 \%$, 


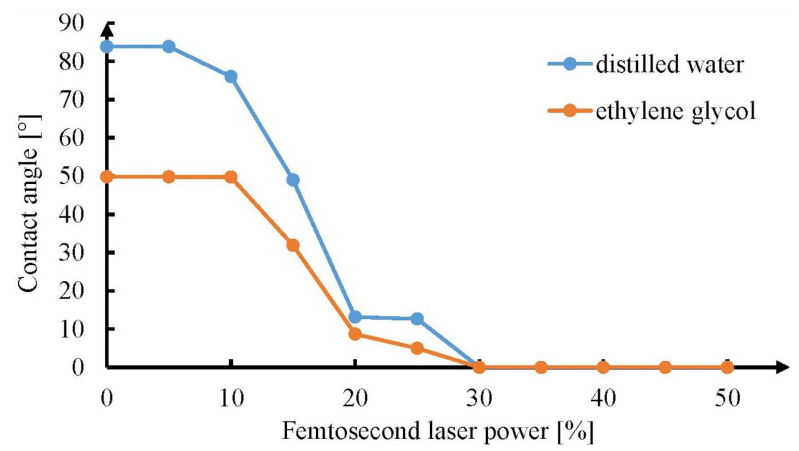

Fig. 1. Distilled water and ethylene glycol contact angle of treated surface with different femtosecond laser power.

the surface activation process is not present. The contact angle does not changing then, or neither the surface free energy. On this level there is not enough energy from the laser beam in order to change the surface boundary layer to polar, changing the functional molecule groups. The first significant improvement of the wettability is when $15 \%$ average laser power was used, namely, both the distillated and ethylene glycol contact angle decreased, the surface energy increased, overall resulting in better adhesion properties on the metallic glass. Using 30\% average femtosecond laser power the activated areas changed into hydrophilic state, both contact angles were $0^{\circ}$, and $0.88 \mathrm{~N} / \mathrm{m}$ surface free energy could be reached - according to Fowks surface theory. Until $15 \%$ power was used there was no visual changes on the specimen. A weak visual modification appeared under $20 \%$ laser power, however using $40 \%$ power a significant visual difference could be observed.

\subsection{The material structure results of the treated surfaces}

In order to examine the structural changing effect of laser five specimen were investigated: the specimens with $0 \%, 15 \%, 20 \%, 40 \%$, and $50 \%$ average laser power treated. The results can be seen in Fig. 2.

Optical microscope images of the laser irradiated metallic glass samples showed the change in surface morphology that strongly depend on the average power of laser. Figure 2a determines the original, untreated surface. As shown in Fig. 2b, the topography of treated specimen is changed a little, while the path of the laser scanning area is hardly seen. This is the limit where the laser starts to remove, or melt the material during the activation process. In Fig. 2c, when $20 \%$ average power was used there is a significant changed in the topography. The laser scanning left the parallel grooves in the surface. When the wettability changes to hydrophilic using $40 \%$ average laser power (Fig. 2 d), it indicates that the femtosecond laser beam vaporizes the base material after the photon absorption, and causes a plasma blast in the boundary layer. These modifications are clearly
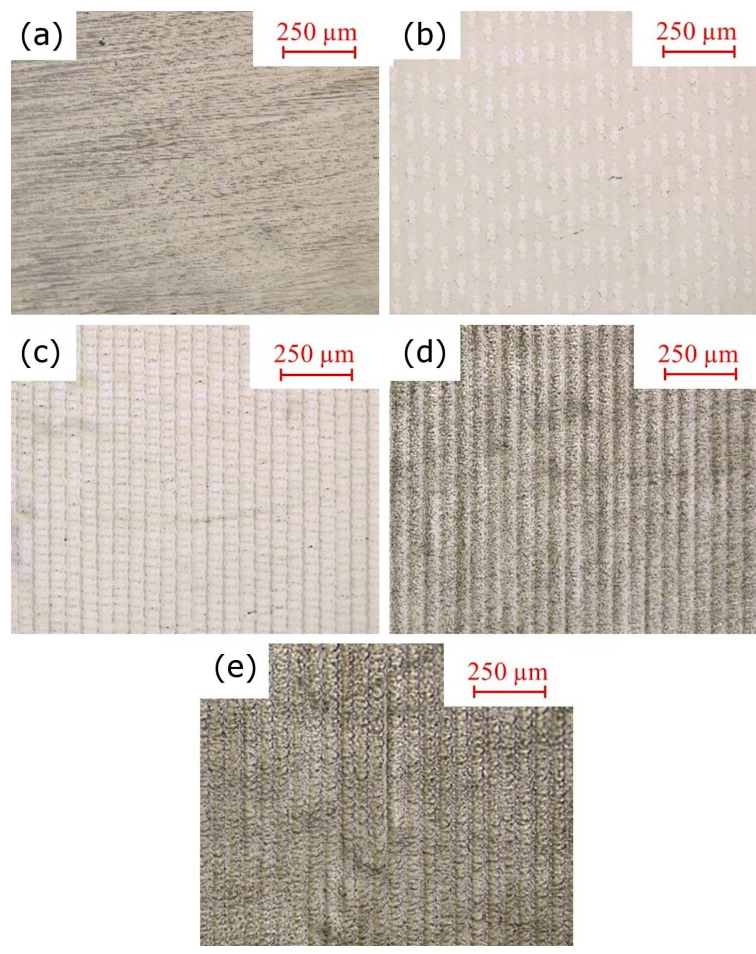

Fig. 2. Optical microscope images of the femtosecond laser treated metallic glass specimens (a) 0\%, (b) 15\%, (c) $20 \%$, (d) $40 \%$ and (e) $50 \%$ average laser power.

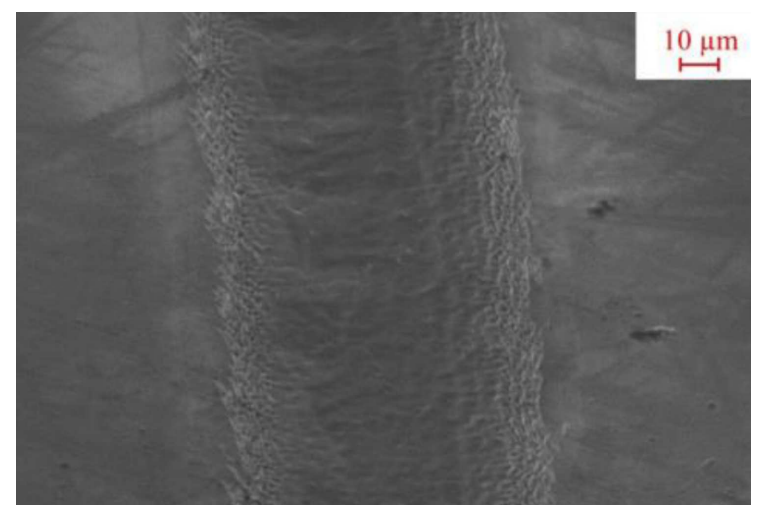

Fig. 3. SEM image of the metallic glass surface after one path femtosecond laser treatment with a $40 \%$ average power.

visible on the metallic glass, however the specimen does not become any rigid, but it keeps its flexibility. Using $50 \%$ average or even higher laser power the surface became damaged heavily and the structure became to very rigid, as shown in Fig. 2e. As Fig. 3 shows the use of $40 \%$ average laser power causes a significant groove on the surface where the center of the treated path is smooth, in the heat effected zone where the laser beam intensity lowered the surface is become bumpy. The material evaporated during the laser process. The SEM chemistry analysis does not show significant changes between 


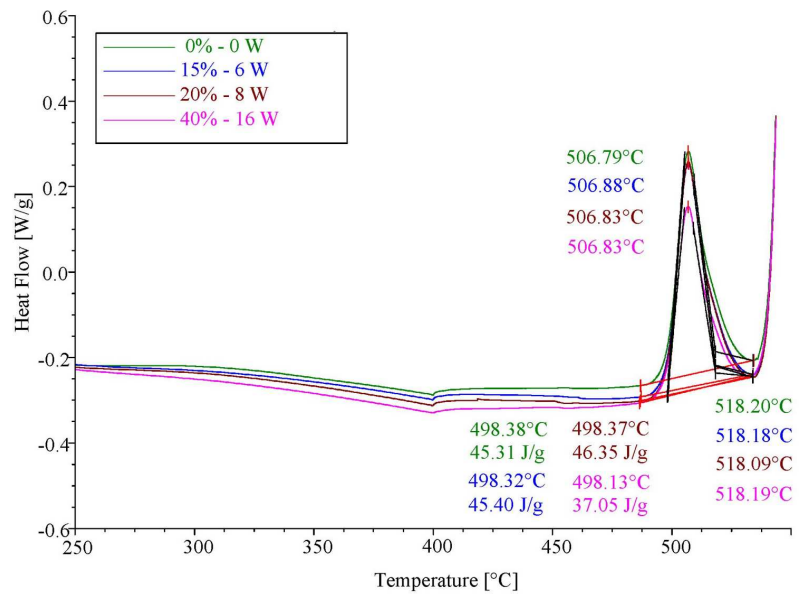

Fig. 4. DSC measurement results of untreated and laser surface treated metallic glass specimens.

the base material and the center of the laser line path using $40 \%$ average laser power. On the side of the path laser-induced periodic surface structures can be seen. These surface topography changing should be avoided, these can cause the changing in magnetic results.

The DSC measurement shown no significant differences between the structure of base material and $15 \%$ and $20 \%$ laser surface activated samples, only the high $40 \%$ samples was significant different. The enthalpy change of the $40 \%$ average laser power treated sample was $19 \%$ lower than the base material and the lower power treated samples (Fig. 4.). It suggests that the topography and the structure of the $40 \%$ sample was modified.

\subsection{The magnetic test results of the treated samples.}

For the magnetic testing of the metal glass 4 type of $100 \mathrm{~mm} \times 10 \mathrm{~mm}$ specimens were made, as shown in Fig. 5. The magnetic test results are given in Table I, and the magnetization curves are summarized in Fig. 6 .

The laser surface treatment have negative effect on the magnetic properties. The coercive force, the demagnetizing factor, the anisotropic and the tension anisotropic constant increase, while the magnetic permeability of magnetic particles decreases. Using on and below $20 \% \quad(8 \mathrm{~W})$ of average power of the femtosecond

\section{TABLE I}

Magnetic properties of untreated and femtosecond laser surface treated metallic glass samples.

\begin{tabular}{l|c|c|c|c}
\hline \hline Laser power [\%] & 0 & 15 & 20 & 40 \\
\hline$J_{s}[\mathrm{~T}]$ & 1.499 & 1.489 & 1.485 & 1.499 \\
$H_{c}[\mathrm{~A} / \mathrm{m}]$ & 4.27 & 7.5 & 8.95 & 11.43 \\
$K_{i}\left[\mathrm{~J} / \mathrm{m}^{3}\right]$ & 471.3 & 523.9 & 712.4 & 1143 \\
$K_{\sigma}\left[\mathrm{J} / \mathrm{m}^{3}\right]$ & 434.2 & 493.7 & 655.3 & 1106.4 \\
$D \times 10^{5}$ & 8.56 & 10.23 & 14.2 & 10.5 \\
$\mu_{p} \times 10^{-3}[\mathrm{H} / \mathrm{m}]$ & 3.298 & 3.011 & 2.521 & 1.565
\end{tabular}

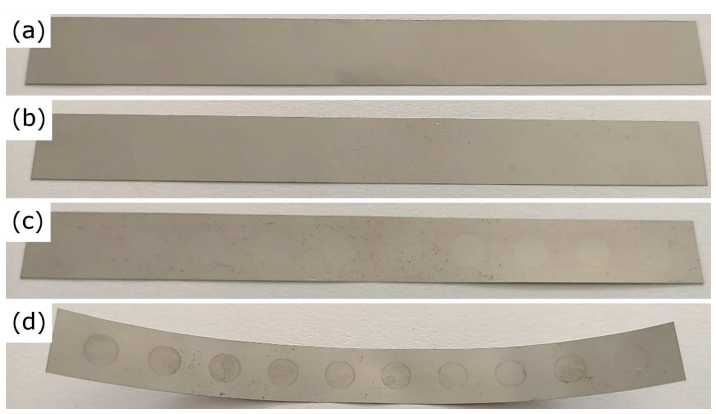

Fig. 5. Surface activated metallic glass samples treated with (a) $0 \%$, (b) $15 \%$, (c) 20\%, and (d) $40 \%$ average power of femtosecond laser.

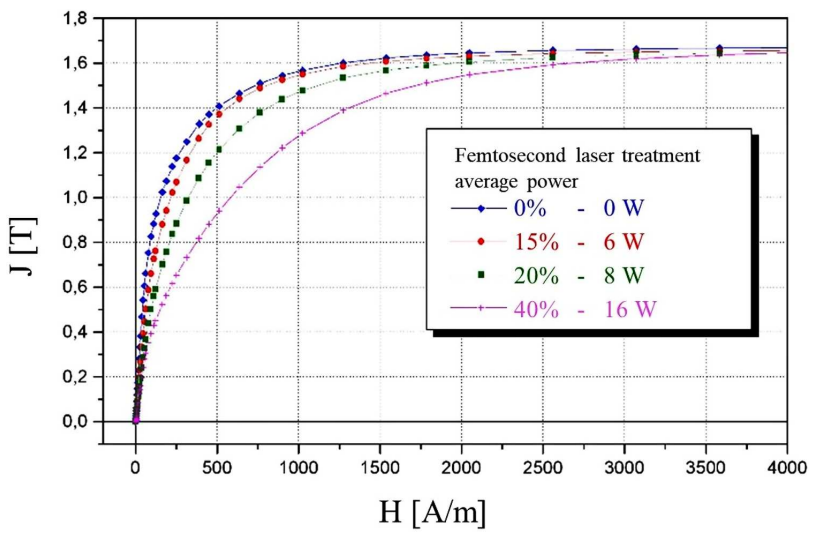

Fig. 6. Magnetization curves of untreated and femtosecond laser treated metallic glass samples.

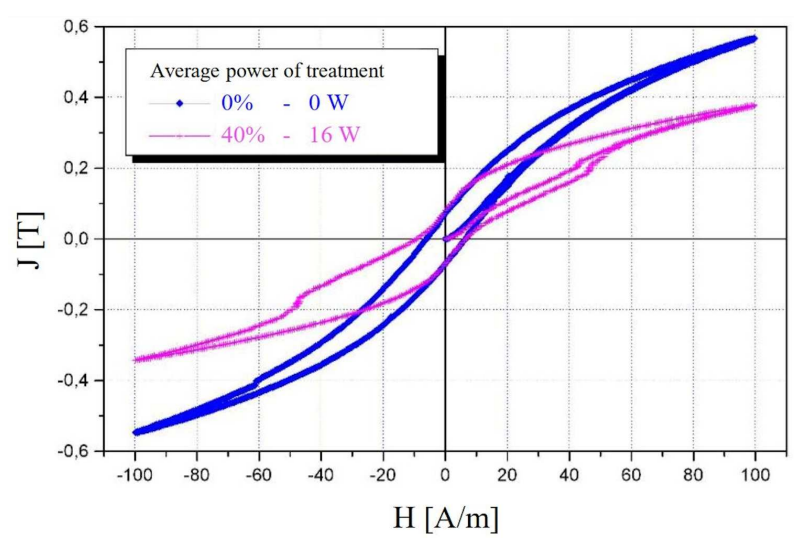

Fig. 7. Magnetization curve of base material and $40 \%$ average laser power treated samples.

laser, its the cold impulses change only the magnetic properties with a lower ratio because only a few micrometer of the structure changes. In the activated surface zones there are both good and wrong magnetic properties of the metallic glass. The reason for the change is examined in the Fig. 7 . 
The magnetic properties of sample treated a high power laser and the sample untreated one, are very different, see Fig. 7. The difference is noticeable between two magnetization curves, while the most deformed curve corresponds to the $40 \%$ laser treated. The magnetization curves were measured with the resolution of $0.1 \mathrm{~A} / \mathrm{m}$. During the process the laser beam is able to activate the surface of the metallic glass on the one of its side only. Thus, not the entire volume of the specimen causes the effect, but only a thin layer which is irradieted.

\section{Conclusions}

In this work the femtosecond laser surface activation of the FINEMET type metallic glass was investigated. Four types of specimen were investigated: one untreated with laser power and other three treated with 15\%, $20 \%$ and $40 \%$ average laser power. Using a laser surface activation, the hydrophilic state of the metallic glass specimens was able to reached. The topography and structural properties introduced rather a little difference from the base material. During metallography examinations only significant changes appeared in the $40 \%$ or higher power treatment. In case of the magnetic properties of the samples they were always changing and were damaged because the laser treatment depends on the laser power. The wetting properties could be increased using the developed surface activation method, which opens up door of new opportunities for the electronics industry.

\section{Acknowledgments}

The project has been supported by the European Union, co-financed by the European Social Fund. EFOP3.6.1-16-2016-00014. I thank Ladislav Novak for the assistance in the magnetic measurements.

\section{References}

[1] L. Hubac, L. Novak, S. Balla, A. Lovas, Acta. Phys. Pol. A 131, 672 (2017).

[2] B. Vehovszky, N. Ruzsás, J. Takács, Z. Weltsch, Peri. Polyt. Trans. Eng. 41, 105 (2013).

[3] Z. Weltsch, A. Lovas, J. Takács, Á. Cziráki, A. Toth, G. Kaptay, App. Surf. Sci. 268, 52 (2013).

[4] M. Lisnichuk, Yu. Katuna, K. Saksl, V. Girman, J. Gamcová, D. Balga, M. Durišin, J. Kováč, P. Kollár, P. Sovák, Acta. Phys. Pol. A 131, 672 (2017).

[5] A. Szabo, R. Varga, F. Ujhelyi, V. Komanicky, A. Zorkovska, A. Lovas, Acta. Phys. Pol. A $\mathbf{1 2 6}$ 204 (2014).

[6] L. Novak, J. Kovac, L. Hubac, Acta. Phys. Pol. A 131, 753 (2017). 